Vera Kalitzkus (2009): Als Ethnologin in der Medizin? Ein persönliches Resümee. In: Elfriede Hermann, Karin Klenke und Michael Dickhardt (Hg.): Form, Macht, Differenz. Motive und Felder ethnologischen Forschens. Göttingen: Universitätsverlag Göttingen, 347-355. Doi: $10.17875 /$ gup2020-1296

\title{
Als Ethnologin in der Medizin? Ein persönliches Resümee
}

\author{
Vera Kalitzkus
}

Wann ist eine Ethnologin eigentlich eine Ethnologin? Sie hat das entsprechende Fach studiert, dort auch promoviert - und dann? Viele betätigen sich danach in anderen Berufsfeldern und akademischen Disziplinen. Ich bin jetzt seit kurzem in der Allgemeinmedizin gelandet: als wissenschaftliche Mitarbeiterin am Institut für Allgemeinmedizin und Familienmedizin der Universität Witten/Herdecke. Über ein Jahrzehnt nach Beginn des DFG-Forschungsprojektes von Brigitta Hauser-Schäublin zu „Verwandtschaft und Humantechnologie: Die kulturelle Dimension von Reproduktionsmedizin und Organtransplantation" im Jahre 1996 ist dies ein guter Zeitpunkt, um den zurückgelegten Weg zu reflektieren.

Bin ich noch Ethnologin? Und wenn ja, woran merkt man das? Merke ich es noch? Oder habe ich ethnologisch gesehen in den letzten Jahren den Weg des going native beschritten in andere Fachdisziplinen und Wissenstraditionen (etwa der medizinischen Psychologie/Soziologie, der Medizintheorie und der allgemeinmedizinischen Forschung)? Denn die Projekte, in denen ich mitarbeitete, verfolgten keine ethnologische Fragestellung und waren auch nicht interdisziplinär angelegt; auch war ich - mit Ausnahme meiner jetzigen Station in der Allgemeinmedizin - nicht aufgrund meines ethnologischen Hintergrunds beteiligt, sondern wegen meiner Erfahrung in qualitativer Forschung und dem Arbeitsschwerpunkt Patientenperspektive/Umgang mit schweren Erkrankungen. 
Das damalige Projekt „Verwandtschaft und Humantechnologie“ zum Umgang mit neuen medizinischen Technologien in Deutschland erkenne ich rückblickend als Weichenstellung in meinem Werdegang - beruflich wie persönlich. Es hat mich aus der „klassischen“ Ethnologie hinaus- und in den medizinischen Bereich hineingeführt. Seither war und bin ich mit dort angesiedelten Projekten zu Krankheitsverarbeitung, Patientenversorgung, Gesundheitsförderung oder Arzt-Patienten-Kommunikation beschäftigt. Das könnte nun auch explizit kulturelle Aspekte umfassen, wie etwa die Krankheitsvorstellungen bestimmter Migrantengruppen, transkulturelle Psychiatrie oder andere interkulturelle Aspekte im Gesundheitswesen. Doch das ist nicht der Fall. Was also mache ich dort, bin ich überhaupt noch Ethnologin?

Die Themen und Projekte, mit denen man beschäftigt ist, hinterlassen Spuren. Die Promotionszeit kommt einer langen Initiationsphase in die Identität als Ethnologin und wissenschaftlich Arbeitende gleich; sie ist nie nur rein intellektuelles Abenteuer. Für mich war sie verbunden mit einem persönlichen Wachstumsprozess, in dem die Fragen nach den eigenen Zielen und Werten im Leben, die sich auch in meiner Arbeit niederschlagen sollen, von zentraler Bedeutung waren. Dieser Aufsatz ist der Versuch, den Weg durch diese Zeit bis zu meiner heutigen Tätigkeit zu reflektieren - eine Art persönliches Resümee, das vielleicht zur Reflexion über das eigene Selbstverständnis anregen kann.

\section{Ethnologin sein in der eigenen Gesellschaft}

Brigitta Hauser-Schäublin fragt in ihrem Aufsatz „Blick zurück im Zorn: Ethnologie als Kulturkritik“ (1997) nach Grenzen und Territorien der Ethnologie und was von ihr übrig bleibt, wenn sie sich auch komplexen Gesellschaften, gar der eigenen Gesellschaft zuwendet. „Ist sie dann keine autonome Wissenschaft mehr, sondern nur eine besondere Methode, die andere Wissenschaften nach Bedarf anwenden?", zitiert sie Stagl (1981:36) in diesem Aufsatz. Anders stellt sich die Frage, wenn man sich dazu auch noch aus der Disziplin selbst entfernt. Wie viel bleibt von meiner Ethnologen-Identität noch, wo ich doch sowohl thematisch als auch institutionell in "fremden Territorien“ arbeite?

Nicht nur einmal habe ich gehadert mit dem Promotionsprozess im Allgemeinen und der Forschung zum Thema Organtransplantation im Besonderen. Die Kommilitoninnen und Kommilitonen aus dem Doktorandenkolloquium kehrten zurück aus Fidschi, Papua Neuguinea, Guatemala oder Indonesien - nicht immer braun gebrannt und glücklich, aber immerhin waren sie weg gewesen, in fremden Welten und fernen Ländern! Und ich? Ich konnte stattdessen von Kaffee und Kuchen bei Selbsthilfegruppen Organtransplantierter erzählen, dramatischen und schwer zu ertragenden Szenen auf der Intensivstation einer großen Klinik oder von Leid und Elend auf einer Transplantationsstation. „Highlights“ gab es zwar auch - etwa eine im OP live mit- 
erlebte Transplantation oder die Begleitung der deutschen Sportmannschaft zu den World Transplant Games in Sydney, Australien. Das mutete mir auch recht „exotisch“ an, aber leider nicht so, wie man sich das gemeinhin von der Ethnologie erträumt: also der romantische Traum einer Feldforschung irgendwo in der exotischen Fremde, was eh einer unzulässigen Einengung des Ethnologischen auf diesen Bereich gleich käme.

Einige Probleme, vor denen meine Kolleginnen aus dem Projekt und ich uns in dieser Feldforschung sahen, sind vergleichbar mit denen in einem „fremden“ Land: Zugang finden zum Feld, Vertrauen schaffen, die eigene Rolle definieren, die medizinische Sprache verstehen, Tabuthemen erkennen. Auch gilt als akzeptiert, dass die traditionellen Fachgrenzen nicht mehr tragen und die neue komplexe vernetzte Welt nur inter- oder transdisziplinär angegangen werden kann und somit auch unser Thema ein ethnologisches war. Trotzdem waren wir irgendwie „draußen“ damit. Als Initiation in den Reigen der „,richtigen“ Ethnologen zählte nach wie vor nur die „echte“ Feldforschung, so wurde uns in vielen Reaktionen auf unser Projekt deutlich.

Setzt man als ausschlaggebendes Kriterium aber die Fremdheitserfahrung, das BeFremdetsein im Alltäglichen - es hätte in der Zeit meiner empirischen Erhebung nicht intensiver sein können. Das Eintauchen in ein neues Feld geht einher mit dem Wagnis, tiefe eigene Überzeugungen dessen, wie die Welt ist, und wie man sich selbst versteht, in Frage zu stellen. Davor schützt auch die Forschung in der eigenen Gesellschaft nicht. Mehr noch: die Be-Fremdung des Eigenen findet ja zuhause statt - ohne die Hoffnung auf einen rettenden Heimflug dorthin, wo alles so ist, wie wir es kennen (auch wenn diese Erfahrung durch ein etabliertes soziales Netzwerk und Gesprächspartner zu Hause aufgefangen werden kann).

\section{Im „Feld“ der Transplantationsmedizin}

Feldforschung berührt immer die ganze Person. Sie birgt in der eigenen Gesellschaft andere Herausforderungen als in einem fremden Land. „Die kulturelle Distanz ist über weite Strecken eine Voraussetzung für das ,Sehen' von nahezu Ausgeblendetem, aber auch von routinisierten Abläufen, von standardisiertem Verhalten und als normal geltenden Ansichten und Gefühlen“" schreibt Brigitta Hauser-Schäublin (2003:38). Diese Distanz muss man sich in der eigenen Gesellschaft immer wieder bewusst heranziehen, eine „ethnologische Brille" aufsetzen, durch Erinnerung an die eigene Erfahrung in "fremden" Kulturen und die ethnologischen Theorien und das Wissen um andere kulturelle Konzepte zu den Themen, die man in der eigenen Gesellschaft untersuchen möchte.

Bei mir ging es um die scheinbar so einfach zu beantwortende Frage, wann ein Mensch tot ist und nicht mehr zur Gemeinschaft der Lebenden dazugehört. In einem Gespräch mit Brigitta Hauser-Schäublin darüber, was die teilnehmende Beobachtung 
in der eigenen Gesellschaft zum Thema „Organtransplantation“ für mich bedeutete, reflektierte ich:

Teilnahme bedeutete für mich in diesem Kontext die Konfrontation mit Krankheit und Tod. Da es sich um ein gesellschaftlich stark umstrittenes Thema handelt, das ich beforschte, wurde ich zudem immer wieder von meinen GesprächspartnerInnen vor das Problem gestellt, Position zu beziehen. Ich denke, es ist in einer fremden Gesellschaft einfacher, sich vorzubehalten, eine eigene Position zu beziehen. In der eigenen Gesellschaft ist die Frage nach der eigenen Verortung sehr viel schwieriger. [...] Ich habe gemerkt, wie schwer es ist, die Schicksale der Menschen, mit denen ich sprach, mir vom eigenen Leib zu halten, mir nicht zu sehr unter die Haut gehen zu lassen - gerade, weil es meine Aufgabe war, direkt einzutauchen in die Lebenswelt der Betroffenen. (Kalitzkus zitiert in Hauser-Schäublin 2003:39-40)

Die Herausforderung an mich, Position zu beziehen, wurde dadurch erschwert, dass sich das Feld „Transplantationsmedizin“ durch starke Polarisierungen auszeichnet: zum einen zwischen Befürwortern und Kritikern der Transplantationsmedizin, zum anderen zwischen der Seite der Angehörigen von Organspendern und der Seite der Organempfänger. „Teilnahme bedeutet Nähe, Beobachten Distanz: Teilnehmende Beobachtung setzt sich deshalb aus widersprüchlichem Verhalten zusammen, nämlich so zu sein, wie einer, der dazu gehört und gleichzeitig mit einer Wahrnehmung wie einer, der außerhalb steht.", schreibt Brigitta Hauser-Schäublin (2003:38, Hervorhebung im Original). Das kann zu einer seelischen Zerreißprobe werden, wenn die unterschiedlichen Gruppen zum Teil miteinander unvereinbare Positionen und Interessen vertreten, bei denen es noch dazu um Leben oder Tod geht. Mir tat sich eine Welt voller schwer zu ertragender Ambivalenzen auf, die mich immer wieder zwang, mich zu offenbaren: „Bist Du dafür oder dagegen?““. Und damit auch: „Bist du für oder gegen uns?“, ja sogar „Sprichst Du uns damit das Recht auf Leben ab?" Der Wechsel zwischen der spendenden und der empfangenden Seite fiel mir besonders schwer. Doch diese äußerst unangenehmen Empfindungen wiesen mich auch auf ein Kernproblem der Transplantationsmedizin hin: das Überleben auf der Empfängerseite ist mit Tod, Trauer und Schmerz auf der Spenderseite verbunden. Die Angst vor dem Tod, das Verzweifeln am Tod lässt die Diskussion um Organtransplantationen so heftig werden (vgl. Kalitzkus 2003). Das ethnologische Unterfangen scheint per se zu bedeuten im „Dazwischen“ zu sein und darüber Erkenntnisse zu erlangen, wie in einem späteren Abschnitt noch weiter ausgeführt wird. Ich kann mir kaum einen schmerzlosen Weg vorstellen, um dieses Abenteuer zu überstehen. 


\section{Ethnologie als Kulturkritik - Das Beispiel Transplantationsmedizin}

Die Ergebnisse unserer Studie kritisch reflektiert in die Diskussion der eigenen Gesellschaft einzubringen, das war Ziel unseres Projektes, wie Brigitta Hauser-Schäublin auch im „Blick zurück im Zorn“ schreibt (1997:10): dass wir als Ethnologen Stimmen wahrnehmen und sie auch zu Worte kommen lassen, die mit der dominierenden Meinung und dem sogenannten allgemeinen Konsens nicht übereinstimmen, oder gar ungehört verklingen. Diese Stimmen „hört“ man natürlich aus einem bestimmten Grund, aufgrund der Werte und „Positionierung“ im Feld. Es ist wichtig, „[...] dass der Standort des Ethnologen, seine Werteposition für die Auswahl der Stimmen und der Art ihrer Präsentation verantwortlich ist. Diese Position wird in jedem Fall bezogen - aber es gilt auch, sie sichtbar zu machen und zu vertreten“" (Hauser-Schäublin 1997:10; vgl. auch Kalitzkus 2005).

Sich der gesellschaftlichen Diskussion zu stellen, zumal im Kontext der Transplantationsmedizin, kann recht „ungemütlich“ sein, insbesondere dann, wenn man unpopuläre und unbequeme Ansichten vertritt: etwa zur Auseinandersetzung mit dem Tod, der „Hirntod“-Debatte, zur Bedeutung des verstorbenen Körpers für Abschiednehmen und Trauer, oder der Frage nach dem Verständnis vom Menschen und den Werten, mit denen es verbunden ist. Denn wir wissen aus ethnologischen Studien, dass die Elemente, die zur Konstituierung einer Person gehören, häufig „über die materielle Beschaffenheit des Körpers, seine Vollständigkeit/Unvollständigkeit und seine Disposition z. T. weit hinaus [gehen] und sich mit der sozialen Einordnung des Individuums in Gruppen sowie mit transzendentalen Dimensionen [befassen] " (Hauser-Schäublin 1997:12). Die Transplantationsmedizin möchte von einem solchen komplexen Bild des Menschen nichts wissen. Brigitta Hauser-Schäublin stellte 1997 die Hypothese auf, die Transplantationsmedizin versuche, „sich frei vom Faktor verwandtschaftlicher Beziehungen, den Körper, Körpersubstanzen und -teile symbolisieren, zu halten, um diese Errungenschaften auch möglichst frei und ungebunden einsetzen zu können, daß aber das, was das Resultat davon ist, der Warencharakter, den diese Körpersubstanzen durch deren Integration in einen internationalen Markt erhalten, unsichtbar gemacht oder verhüllt wird“ (Hauser-Schäublin 1997:15). Das hat sich leider bestätigt, die Tendenz der fortschreitenden Kommerzialisierung des Körpers ist eindeutig, wie wir auch in der aktualisierten neuen Auflage unserer Projektpublikation „Der geteilte Leib“ (2008) darlegten. Die Stimmung in der öffentlichen Diskussion hat sich in jüngster Zeit erneut verschärft: „Organspende“ wird mehr und mehr zur moralischen Pflicht, die Beteuerung, jede Einstellung werde akzeptiert, verkommt zu einem Lippenbekenntnis. Sogar der Nationale Ethikrat behauptet in seiner Stellungnahme zur Organspende aus dem Jahr 2007: „Die Bereitschaft zur postmortalen Organspende ist ethisch als die objektiv vorzugswürdige Alternative anzusehen“ (Nationaler Ethikrat 2007:29, Kalitzkus 2009). Eine Studie allein kann an einer gesellschaftlichen Entwicklung wenig ändern. Was bleibt, ist zumindest ein Korrektiv anzubieten für die begrenzte Sichtweise auf 
Gesundheit und Heilung, wie sie im dominanten Paradigma der Biomedizin vorherrsche (Hare 2001:305).

\section{Was kann für die Medizin hilfreich sein?}

Ethnologische Themenfelder in der Medizin gibt es viele: sei es die medizinische Versorgung von Migranten und Flüchtlingen, das Hinterfragen medizinischer Konzepte, die „kulturelle Gebundenheit“ medizinischen Wissens, die Analyse der „Mikrokosmen“ in Krankenhäusern und Kliniken, aber auch in der Hausarztpraxis. Die Betreuung von Menschen mit anderem ethnischen Hintergrund gehört auch in Deutschland zum Alltag medizinischer Praxis. Längst gibt es schon Tagungen, die dieses Thema direkt von und für praktizierende Ärzte anbieten. Doch alles Wissen um interkulturelle Medizin, kulturelle Sensibilität, Kontextualität von Vorstellungen zu Körper, Gesundheit und Krankheit hilft nicht viel, wenn dem Arzt oder der Ärztin im direkten Kontakt mit den Patienten - egal ob deutsch, türkisch oder vietnamesisch - der Mut zur Begegnung fehlt.

Der zurückgelegte Weg hat in mir eine Vorliebe hinterlassen für Themen, die von individueller Entwicklung zeugen und Projekte, die danach streben, die (medizinische!) Welt ein wenig menschlicher zu gestalten - im Sinne einer stärkeren Orientierung an den Bedürfnissen der Patienten, aber auch im Sinne einer lebensgerechteren Arbeitswelt für die Gesundheitsberufe. Das hat immer mit Begegnungen zwischen Menschen zu tun und der Achtung vor ihrer jeweiligen Erfahrung. In der Medizin kann eine solche Haltung durch den Ansatz der narrativen Medizin entstehen, einer Richtung, die den Wert von Erzählungen als basales Instrument unseres Selbst- und Weltverständnisses sowie der Beziehungsbildung wieder in den Mittelpunkt rückt und die ein Weg sein könnte, die medizinische Welt und ihre alltägliche Praxis menschlicher zu gestalten (Kalitzkus et al. 2009).

\section{Sich selbst als Erkenntnisinstrument begreifen}

Den eben skizzierten Ansätzen ist zu Eigen, dass sie die Reflexion über die eigene Position und Erkenntnis erfordern. Die Ethnologie ist eine Wissenschaft des Verstehens, so Brigitta Hauser-Schäublin, und eine Disziplin des „Dazwischenstehens“. Sie zitiert Hans-Georg Gadamer (1990:300, nach Hauser-Schäublin 1997:6): „In diesem Zwischen ist der wahre Ort der Hermeneutik [...] Aus der Zwischenstellung, in der die Hermeneutik ihren Stand zu nehmen hat, folgt, dass ihre Aufgabe überhaupt nicht ist, ein Verfahren des Verstehens zu entwickeln, sondern die Bedingungen aufzuklären, unter denen Verstehen geschieht." Das ist auch ein zentrales Anliegen in der Medizin und der Forschung zur Arzt-Patienten-Kommunikation. Zentral ist dabei das Subjekt, 
das das Abwägen und Verstehen vornimmt - es ist das Verbindungsglied zwischen dem Fremden und dem Eigenen, schreibt Brigitta Hauser-Schäublin weiter: „Diese Rolle des Dazwischenstehens spielt der Ethnologe als Subjekt mit allen seinen Prägungen und Idiosynkrasien, aber auch mit seinem Wissen, ein Subjekt, das indirekt, durch das Studium des Fremden, auch sich selber studiert" (Hauser-Schäublin 1997:7). Und durch dieses Selbststudium können Erkenntnisse erlangt werden, die auch etwas über das beforschte Thema aussagen. Beobachtung und Lernen seien dabei wichtige Faktoren, „weil sie zur Objektivation und zur Sichtbarmachung von Kultur führen“ (Hauser-Schäublin 1997:7).

Dieses Verständnis ist auch in der Medizin von zentraler Bedeutung. Denn neben den Erkenntnissen aus breit angelegten randomisiert-kontrollierten Studien, die der Goldstandard in der evidenzbasierten Medizin sind, gilt es immer, dieses Wissen auf den konkreten einzelnen Patienten mit seiner einzigartigen Geschichte zu beziehen (Matthiessen 2006). Sich selbst mit allen Sinnen als Erkenntnisinstrument zu begreifen - und zwar nicht nur den eigenen Verstand, sondern auch körperliche Empfindungen und Gefühle, über die man wichtige diagnostische Hinweise bekommen kann, das ist ein Anliegen, wie ich es Medizinstudierenden zu vermitteln versuche. Erstaunlich ist, wie viele Fähigkeiten, die eine gute Feldforscherin auszeichnen, auch wichtige Merkmale für einen guten Arzt sind: „Teilnehmende Beobachtung verlangt vom Ethnologen, dass er seine Aufmerksamkeit schärft, sozusagen alle seine Sinne ausführt, um auch Dinge wahrzunehmen, die sonst nicht wahrgenommen werden" (Hauser-Schäublin 2003:37). Spittler spricht dabei von „dichter Teilnahme“: „Zu diesem Erleben gehören alle Sinne, nicht nur das Sehen und Hören, sondern auch das körperliche und seelische Fühlen" (Spittler 2001:19, zitiert nach Hauser-Schäublin 2003:38; Hervorhebung im Original). Robert Hahn, Arzt und medical anthropologist beschreibt als anzustrebende Prinzipien in der Medizin etwa aktives und tiefes Zuhören; Kontextverstehen; intraethnische Unterschiede erkennen; erklären, übersetzen und vermitteln können (Hahn 1995:274-288). Diese Aufzählung ist fast identisch mit den wichtigsten Merkmalen, die der Hausarzt John Launer (2002) für den Ansatz einer narrativen Medizin nennt.

\section{Ausblick}

Was ich als wertvolles Gut aus meiner Zeit in der ,richtigen“ Ethnologie mitnehme, das ist das Wissen um die menschliche Vielfalt, ein komplexes Menschenbild, die Bedeutung von Subjektivität in der Wahrnehmung dieser Welt und die persönliche Erfahrung des Dazwischenseins. Der unglaubliche Erfindungsreichtum der Menschen, sich die Welt und den Kosmos zu erklären: die „natürlichen“ Gegebenheiten mit Bedeutung zu versehen; den Körper als Ausdruck für Gefühle, familiäre oder soziale Konflikte zu nehmen; den Körper als Wahrnehmungs-, in gewisser Weise aber auch Ausdrucksinstrument für das Leben mit all seinen Facetten zu erkennen; die sich unterscheidenden Arten und 
Weisen, wie sich Welt, soziale Gemeinschaft und individuelle Vorstellungen im Körper und den leiblichen Empfindungen niederschlagen. Meinen „ethnologischen“ Beitrag sehe ich darin, für die Vielschichtigkeit und Komplexität menschlichen Lebens zu sensibilisieren, die gerade in der modernen Medizin so schnell aus dem Blick gerät. Doch diese Komplexität muss ich auch mir selbst immer wieder bewusst machen: durch stetige Rückbesinnung, Eintauchen in ,andere“ Welten (und sei es „nur“ durch Bücher), um immer wieder frisch durch die „ethnologische Brille“ auf unsere Gesellschaft und ihr medizinisches System zu blicken. Dazu gehört auch Selbstdisziplin, um sich immer wieder in den (zumindest inneren) Zustand des ungemütlichen „Dazwischen“ zu versetzen. Diesen Artikel zu schreiben, hat mir dies erneut deutlich vor Augen geführt. Es zeigt mir den Wert, den das „Dazwischen“ hat, auch wenn es im Arbeitsalltag oftmals eine Gratwanderung ist.

Mein bisher zurückgelegter Weg war nicht immer einfach, missen aber möchte ich ihn nicht. Ich bin dankbar für die Herausforderung, vor die mich diese Erinnerung an den Perspektivwechsel immer wieder stellt; dankbar dafür, dass ich meine Arbeit als Chance zu innerem Wachsen begreifen kann. So hat sich mir der Weg durch die verschiedenen Projekte und Disziplinen als innerer Werdegang, Formung meiner beruflichen Identität und meines Selbstverständnisses als Forschende gezeigt: als Fragende zum Thema des menschlichen Umgangs mit Krankheit und Leid und dem Potential zu persönlichem Wachstum.

\section{Literatur}

Gadamer, Hans-Georg (1990) Hermeneutik I: Wabrheit und Methode. - 1. Grundzüge einer philosophischen Hermeneutik. Tübingen: Mohr.

Hahn, Robert A. (1995) Sickness and Healing: An Anthropological Perspective. New Haven: Yale University Press.

Hare, Martha L. (2001) The Promise of Medical Anthropology at the End of the Twentieth Century. Reviews in Anthropology 30(4):291-307.

Hauser-Schäublin, Brigitta (1997) Blick zurück im Zorn: Ethnologie als Kulturkritik. Zeitschrift für Ethnologie 122:3-17.

Hauser-Schäublin, Brigitta (2003) Teilnehmende Beobachtung. In: Bettina Beer (Hg.): Methoden und Techniken der Feldforschung; S. 33-54. Berlin: Reimer.

Hauser-Schäublin, Brigitta, Vera Kalitzkus und Imme Petersen (2008) Der geteilte Leib. Die kulturelle Dimension von Organtransplantation und Reproduktionsmedizin in Deutschland. Überarbeitete Version (2005) und Ergänzung (2008) des gleichnamigen Buches von 2001. Abrufbar unter <http://webdoc.sub.gwdg.de/ $\mathrm{pub} / \mathrm{mon} / 2008 /$ hauser-schaeublin.pdf> [10.9.2009]. 
Kalitzkus, Vera (2003) Leben durch den Tod. Die zwei Seiten der Transplantationsmedizin aus ethnologischer Sicht. Frankfurt am Main: Campus.

Kalitzkus, Vera (2005) Die Bedeutung von Reflexivität und Positionierung im Prozess qualitativer Forschung in der Allgemeinmedizin. Zeitschrift für Allgemeinmedizin $81(6): 243-247$.

Kalitzkus, Vera (2009) Dein Tod, mein Leben. Warum wir eigentlich für Organspenden sind und trotzdem davor zurückschrecken. Frankfurt a.M.: Suhrkamp.

Kalitzkus, Vera, Stefan Wilm und Peter F. Matthiessen (2009) Narrative Medizin Was ist es, was bringt es, wie setzt man es um? Zeitschrift für Allgemeinmedizin 85(2):60-66.

Launer, John (2002) Narrative-Based Primary Care: A Practical Guide. Abingdon: Radcliffe Medical Press.

Matthiessen, Peter F. (2006) Ärztliche Praxis und wissenschaftlicher Status der Medizin. Forschende Komplementärmedizin 13:136-139.

Nationaler Ethikrat (2007) Die Zabl der Organspenden erhöhen - Zu einem drängenden Problem der Transplantationsmedizin in Deutschland. Stellungnabme. Abrufbar unter <http://www.ethikrat.org/stellungnahmen/pdf/Stellungnahme_ Organmangel.pdf $>$ [10.9.2009].

Spittler, Gerd (2001) Teilnehmende Beobachtung als Dichte Teilnahme. Zeitschrift für Ethnologie 126(1):1-25.

Stagl, Justin (1981) Szientistische, hermeneutische und phänomenologische Grundlagen der Ethnologie. In: Wolfdietrich Schmied-Kowarzik und Justin Stagl (Hg.), Grundfragen der Ethnologie. Beiträge zur gegenwärtigen Theorie-Diskussion, S. 1-38. Berlin: Reimer. 